\title{
1 Interest of proton pump inhibitors in reducing the occurrence of COVID-19: a 2 case-control study
}

Frederic Blanc, Cedric Waechter, Thomas Vogel, Benoit Schorr, Catherine Demuynck, Catherine Martin Hunyadi, Maxence Meyer, Denata Mutelica, Nadjiba Bougaa, Samira Fafi-Kremer, Lidia Calabrese, Elise Schmitt, Delphine Imperiale, Catherine Jehl, Alexandre Boussuge, Carmen Suna, François Weill, Alexia Matzinger, Candice Muller, Patrick Karcher, Georges Kaltenbach, Erik Sauleau

\section{Abstract}

Background: COVID-19 is a disease of the elderly as $95 \%$ of deaths related to COVID-19 occur in people over 60 years of age. Despite the urgent need for a preventive treatment there are currently no serious leads, other than the vaccination.

Objective: To find a preventive treatment of COVID-19 in elderly patients.

Design: Retrospective case-control study.

Setting: Robertsau Geriatric Hospital of the University Hospitals of Strasbourg, France.

Patients: 179 elderly patients who had been in contact with the SARS-CoV-2, of whom 89 had tested RT-PCR-positive (COVID-pos) for the virus and 90 had tested RT-PCR-negative (COVID-neg).

Measurements: Treatments within 15 days prior to RT-PCR (including antihypertensive drugs, antipsychotics, antibiotics, nonsteroidal anti-inflammatory drugs, proton pump inhibitors (PPIs), paracetamol, anticoagulant, oral antidiabetics (OADs), corticosteroids, immunosuppressants), comorbidities, symptoms, laboratory values, and clinical outcome were all collected using the electronic patient record.

Results: COVID-pos patients more frequently had a history of diabetes $(P=.016)$ and alcoholism $(P=.023)$, a lower leukocyte count $(P=.014)$ and a higher mortality rate- $29.2 \%$ versus $14.4 \%-$ $(P=.014)$ when compared to COVID-neg patients. Patients on PPIs were 2.3 times less likely (odds ratio $[\mathrm{OR}]=0.4381,95 \%$ confidence interval $[\mathrm{CI}][0.2331,0.8175], \mathrm{P}=.0053$ ) to develop COVID-19 infection, compared to those not on PPIs. No other treatment decreased or increased this risk. COVID-19 patients on antipsychotics $(P=.0013)$ and OADs $(P=.0166)$ were less likely to die.

Limitations: retrospective study.

Conclusion: PPIs treatment lowered the risk of development of COVID-19 infection, and antipsychotics and OADs decreased the risk of mortality in geriatric patients. If further studies confirm this finding, PPIs could be used preventatively in the elderly in this pandemic context. Moreover, OADS and antipsychotics should be tested in clinical trials.

Registration: « Faculté de Médecine de Strasbourg » number CE-2020-68

Funding Source: None 


\section{Introduction}

In November 2019, Wuhan city in China became the centre of an outbreak of pneumonia due to a novel coronavirus (SARS-CoV-2). This disease was named coronavirus disease 2019 (COVID-19) in February 2020 by the World Health Organization (WHO). ${ }^{1}$ COVID-19 is far more dangerous for people aged over 60 , with a death rate of $3.6 \%$ between 60 and 69 years, $8.0 \%$ between 70 and 79 years and $14.8 \%$ after 80 years - and, according to Italian statistics, over $20 \%$ after 90 years -, compared to $2.3 \%$ in the general population. ${ }^{2,3}$ According to WHO, in Europe, $95 \%$ of deaths related to COVID-19 have occurred in people over 60 years of age; ${ }^{4}$ the median age of death for 18176 Europeans was 80 years (range: $24-105) .{ }^{4}$ Furthermore, $91 \%$ of deaths occurred in patients with at least one underlying condition: cardiovascular disease $(42 \%)$, diabetes $(20 \%)$, renal disease $(17 \%)$, malignancy $(17 \%)$, neurological disease including dementia (14\%), and lung disease (11\%). ${ }^{4}$ Chinese data have confirmed that most of the elderly patients who died had multiple comorbidities, and in particular cardiovascular disease (10.5\% mortality), diabetes (7.3\%), chronic respiratory disease $(6.3 \%)$ and hypertension (6\%). ${ }^{2}$ In the United States, obesity appears to be an additional risk factor to other comorbidities. ${ }^{5}$ COVID-19 is therefore an eminently geriatric disease, i.e., it most strongly affects the elderly with multiple comorbidities.

To date, there are no data on treatments that can prevent the development of COVID-19, particularly in the elderly, who are the group most at risk. Therapeutic vaccine trials have begun but even if they are effective, with a favourable risk-benefit balance it is unlikely that we will have access to these vaccines for at least a year. ${ }^{6}$ By then, COVID-19 will have caused major morbidity and mortality. Passive antibody administration through transfusion of convalescent plasma could be a strategy of great interest, but with many challenges. ${ }^{7}$ These limitations are regulatory, logistical and scientific, covering donor eligibility, donor recruitment, collection and transfusion, but also the demonstration of its effectiveness in the elderly population. Thus, a curative rather than a preventive use appears to be more feasible. ${ }^{7}$

Treatments commonly used in the elderly may have a protective effect against COVID-19. Thus, angiotensin 2 AT1 receptor antagonist (angiotensin II receptor blockers [ARBs]) could be of interest to treat or prevent COVID-19. ${ }^{8}$ COVID-19 uses ACE2 as a receptor, a modulator of the activity of different angiotensins ( $I, \|$ and A). The COVID-19-ACE2 interaction increases the activity of angiotensin and thus increases the activity of the AT1 receptor, which results in increased pulmonary vascular permeability and therefore contributes to lung injury. Thus, ARBs could be a protector against lung injury due to SARS-CoV-2, by inhibiting AT1 receptor. ${ }^{9}$ Another avenue of potentially preventive or curative drugs is lysosome-targeted drugs, such as antibiotics, nonsteroidal antiinflammatories (NSAIDs), and proton pump inhibitors (PPIs). ${ }^{10,11}$

In order to look for a treatment that could prevent the development of COVID-19 in elderly patients, we studied the treatments that elderly hospitalized patients were taking before they were tested for COVID-19.

\section{Methods}

\section{Study design and patients}

This was a retrospective case-control study. Elderly patients were considered for the study if they underwent nasopharyngeal swab testing for SARS-CoV-2 between 2 March and 8 April 2020 at Robertsau Geriatric Hospital (University Hospital of Strasbourg [HUS]), Strasbourg, France, including geriatric internal medicine units, follow-up care and rehabilitation units, and long-term care units. Indications for testing were suspicion of COVID-19 because of symptoms consistent with a viral 
infection including viral pneumonitis, contact with COVID-19-infected persons, or presence of COVID19 cases in a hospital unit. The demographic, clinical, biological, and imaging data were retrieved from the professional medical software of the HUS: "DX-care". No patients were excluded as the patient records were all up to date.

We investigated which treatments taken regularly in the 15 days prior to the first swab could have a preventive effect on the disease by analysing the risk associated with each drug. We also looked at the effect of these treatments taken beforehand on survival. If a treatment with an effect on survival was found, we checked whether this treatment was also taken after the first RT-PCR. Patient survival was analysed up to April 17, 2020.

This study was approved by the Institutional Review Board (Ethics Committee of the Faculty of Medicine and University Hospitals of Strasbourg) under number CE-2020-68. Patients were kept informed of our clinical research activity regarding their clinical records via a display in each of the medical units and via an information note given to them upon their arrival.

\section{Data collection}

A manual chart review of randomly selected patients was performed by five investigators (CW, BS, $\mathrm{CMH}, \mathrm{CD}$, and $\mathrm{FB}$ ). Basic demographic and clinical data were extracted, including: medical history and comorbidities, treatment within 15 days prior to SARS-CoV-2 RT-PCR, viral symptoms (cough, fever, dyspnoea, asthenia, or diarrhoea), geriatric syndromes (pain, falls, confusion), pulmonary auscultation, temperature, pulse, blood pressure, weight, height, oxygen saturation (with or without oxygen therapy), clinical outcome (recovery or death), number of SARS-CoV-2 RT-PCRs done for the diagnosis, basic blood results and lung CT scan. Lung CT scan was considered as COVID-19-positive when it demonstrated bilateral or unilateral ground glass opacities or consolidation. ${ }^{12}$ Different treatment of elderly people, including ARBs, angiotensin-converting enzyme inhibitors, diuretics, calcium channel blockers, beta-blockers, statins, drugs for Alzheimer's disease (including rivastigmine, donepezil, galantamine and memantine), drugs for Parkinson's disease (levodopa and dopaminergic agonists), antidepressants (serotonin reuptake inhibitors, serotonin and norepinephrine reuptake inhibitors, tetracyclic, tricyclic), antipsychotics (risperidone, olanzapine, quetiapine, clozapine, haloperidol), anxiolytics (benzodiazepine, zopiclone, zolpidem), anti-epileptics, antibiotics, L-thyroxin, NSAIDs, PPIs, paracetamol, antiplatelet therapies, anticoagulants, oral antidiabetics (OADs), insulin, corticosteroids (oral or inhaled), immunosuppressants, and potassium, were compared between SARS-CoV-2 RT-PCR-positive (COVID-pos) and RT-PCR-negative patients (COVID-neg).

\section{SARS-CoV-2 RT-PCR}

SARS-CoV2 RNA was extracted from nasopharyngeal swabs using the eMAG ${ }^{\circledR} /$ eSTREAM $^{\circledR}$ system (bioMérieux, Marcy l'Etoile, France), then amplified on the LightCycler ${ }^{\circledast} 480$ Instrument II (Roche Diagnostics, France). Quantitative real-time RT-PCR used two targets on the RdRp gene amplified in duplex. ${ }^{13}$ The primers and probes were: Flo2 and Flo4: CoV_IP2-12669Fw ATGAGCTTAGTCCTGTTG and CoV_IP2-12759Rv CTCCCTTTGTTGT with the CoV_IP2-12696Probe (+) AGATGTCTTGTGCTGCCGGTA [5']Hex [3']BHQ-1; CoV_IP4-14059Fw GGTAACTGGGTATGATTTCG and CoV_IP4-14146Rv CTGGTCAAGGTTAATATATAGG with probe CoV_IP4-14084Probe (+) TCATACAAAACCACGCCAGG [5 '] Fam [3'] BHQ-1. They were developed by the national reference 
centre of respiratory viruses at the Institut Pasteur (Paris, France). A standard range of CoV_IP RNA transcript was used for relative quantification. The RT-PCR is specific for SARS-CoV2 and assay sensitivity is around 10 copies/reaction.

\section{Statistical analysis}

The Statistical Package for Social Sciences software (SPSS ver. 22.0.0.0) was used for demographic and clinical data. Differences in these data were assessed using parametric t-tests and for categorical measures $\chi^{2}$ tests were applied. For each test statistic, a probability value of $<0.05$ was regarded as significant.

For exhibiting treatment effect on the probability to have positive RT-PCR, Logistic regression models were used, estimated with Bayesian techniques (McMC, Markov chains and Monte Carlo integration in R statistical software) with the prior assumption that the probabilities of positive RT-PCR in the absence of treatment and in the presence of treatment were of the order of 0.50 . The same kind of model was used with aim to study the treatment impact on mortality adjusting on RT-PCR status. For posterior summaries, median and symmetric $95 \%$ credible intervals were retrieved, and the posterior probability for a parameter to be negative (or positive) was use as a similar quantity than the frequentist $p$-value.

\section{Role of funding source}

There was no funding source.

\section{Results}

By 8 April 2020, 179 patients were suspected of SARS-CoV-2 infection at the Geriatric Hospital of the University Hospital of Strasbourg, France. Among them, 89 were RT-PCR-positive (COVID-pos) and 90 were RT-PCR-negative (COVID-neg). Baseline characteristics and clinical outcomes of COVID-pos and COVID-neg patients are shown in table 1 . The mean age of the 179 patients was 84.06 years (SD 8.20), and all but two were older than 60 years. Most patients were women ( $N=122 ; 68 \%)$. Most patients had contact with COVID-19 at the Geriatric hospital site $(89.9 \%$ for COVID-pos, $77.8 \%$ for COVID-neg). COVID-pos patients more frequently had a history of diabetes $(P=.016)$ and alcoholism $(P=.023)$, a higher frequency of viral compatible symptoms - particularly cough and asthenia $(P<.0001)$, a lower leukocyte count $(P=.014)$ and a higher mortality rate $(29.2 \%)$ when compared to COVID-neg patients (14.4\%) $(P=.014)$. The median length of stay at the hospital site was 8 weeks (SD 82 weeks; minimum $=2$ weeks, maximum $=658$ weeks). The median time from hospital entry to SARSCoV-2 testing was 27 days (SD 567 days; $\min =0$ day, $\max =576$ days). One hundred and twenty-seven patients $(70.9 \%)$ had already been hospitalized for more than 8 days by the time they were tested. The median length of follow-up after first SARS-CoV-2 testing was 25 days (SD 7.46; min=9 days, $\max =39$ days).

Elderly patients on PPIs were 2.3 times less likely (odds ratio $[O R]=0.4381,95 \%$ confidence interval [CI] [0.2331, 0.8175], P=.0053) to develop COVID-19 infection, compared to those with no PPIs (figure 1 and table 2). Forty COVID-neg patients were on PPIs versus 23 COVID-pos patients. The types of PPIs taken by the COVID-neg patients were as follows: pantoprazole $(N=16)$, lansoprazole $(\mathrm{N}=11)$, esomeprazole $(\mathrm{N}=8)$, omeprazole $(\mathrm{N}=4)$, and rabeprazole $(\mathrm{N}=1)$. PPIs taken by the coVID-pos patients were as follows: pantoprazole $(N=15)$, esomeprazole $(N=6)$, lansoprazole $(N=1)$, and omeprazole $(\mathrm{N}=1)$. The median and mean doses for COVID-neg patients were 1.0 (SD .31; $\min =.5, \max =2.0)$ and $.83(\mathrm{SD}=.31)$, respectively, and for COVID-pos patients were .5 (SD .25, $\mathrm{min}=.5$, 
$\max =1.0)$ and $.71(S D .25)$, respectively $(P=.37)$. No other treatment decreased or increased the risk of COVID-19.

The risk of death was lower for COVID-19 patients on antipsychotics $(P=.0013)$ and OADs $(P=.0166)$. It should be noted that no deaths occurred in patients on antipsychotic drugs and only one death occurred in patients on OADs. The types of antipsychotics taken by the 12 COVID-pos patients on antipsychotic drugs were as follows: risperidone $(N=5)$, clozapine $(N=3)$, olanzapine $(N=2)$, alimemazine $(\mathrm{N}=1)$, haloperidol $(\mathrm{N}=1)$. The patient on alimemazine and one patient on olanzapine had these treatments in the 15 days prior to RT-PCR but had their treatment stopped during the COVID-19 infection. All other patients continued their antipsychotic medication during COVID-19 infection. The types of OADs taken by 12 COVID-pos patients on OADs drugs were as follows: metformin $(\mathrm{N}=10$, mean dose $=1310 \mathrm{mg}, \mathrm{min}=500 \mathrm{mg}$, max $=2500 \mathrm{mg}$ ), vildagliptin ( $\mathrm{N}=3$; associated with metformin in one case) and sitagliptin ( $\mathrm{N}=2$, associated with metformin in both cases). Two patients with metformin, including one with sitagliptin, had their treatment stopped during the COVID-19 infection.

\section{Conclusions}

This case-control study aimed to look for treatments that could have a preventive effect on the appearance of COVID-19 in a geriatric population, using data from the main Geriatric hospital of Strasbourg, in the Grand Est region, one of the epicentres of the COVID-19 pandemic in France.

We show for the first time that PPIs could have a preventative effect on COVID-19 infection. Indeed, in our study, taking PPIs was associated with a 2.3-fold reduction in the risk of COVID-19. We did not find that any of the other drugs had a preventive effect on COVID-19. PPIs are among the most prescribed medications for the elderly. Indeed, more than $30 \%$ of people over the age of 80 were reported to take them, ${ }^{14}$ as was the case in this study where $35.2 \%$ were taking PPIs. PPIs are prescribed for acid-related disorders such as peptic ulcer disease and gastro-oesophageal reflux disease (GORD). In our study, out of 63 patients taking PPIs, 31 had a clear indication for this treatment, whereas for the remainder we were unable to ascertain the reason for the prescription, though most of them were also taking antiplatelets or anticoagulants. These drugs are associated with acute lower gastrointestinal bleeding, ${ }^{15}$ and PPIs are effective in treating ulcers. Clinical guidelines generally recommend a PPI treatment duration of less than 8 to 12 weeks. Although usually considered safe, these drugs have been associated with increased risks of side effects such as bone fractures, kidney disease, microscopic colitis, and hypomagnesaemia. ${ }^{14}$ Furthermore, the longterm use of PPIs was recently reported to be associated with an increased mortality risk, ${ }^{16}$ and an increased dementia risk. ${ }^{17}$ PPIs decrease intracellular $\mathrm{pH}$ and increase the extracellular $\mathrm{pH}$ via inhibition of vacuolar $\mathrm{H}^{+}$-ATPases (V-ATPases) in a covalent interaction. ${ }^{18} \mathrm{~V}$-ATPases play a critical role in the maintenance of a neutral intracellular $\mathrm{pH}$ in all cells, and an acidic extracellular $\mathrm{pH}$ by actively excreting protons either through ion exchange mechanisms or by segregating $\mathrm{H}+$ within cytoplasmic organelles that are subsequently expelled. ${ }^{19}$ SARS-CoV-1 and most likely SARS-CoV-2, like other coronaviruses and other enveloped viruses, enter target cells by inducing fusion between viral and cellular membranes. This is mediated by a viral fusion protein named spike of $S$ protein. ${ }^{20}$ The $S$ protein transduction in the cell requires acidification of the endosome or extracellular medium. ${ }^{20}$ Therefore, blocking these receptors inhibits the acidic microenvironment around the cell and in the lysosome, and could prevent the virus from entering the cell. 
212 For this study, we were in quasi-experimental conditions and able to detect COVID-19 infection live 213 since most patients who developed COVID-19 developed it on site, directly inside the geriatric hospital (89.9\%). These are therefore nosocomial infections: presumably through transmission by other patients, through transmission by the paramedical team (nurse, care assistant) or medical team, or, before the introduction of containment measures in France on 17 March 2020, by the families or relatives of the patients. From that date onwards, all visits to the hospital, including those of patients' families, were prohibited. This nosocomial contamination is likely also related to the low use of masks upstream of the contagion, at the beginning of the pandemic in France as there were insufficient stocks, ${ }^{21}$ as well as to the lack of mass testing capability in France, ${ }^{22}$ and more generally to the inexperience and denial of the French population in the face of such a pandemic in its early stages.

The two co-morbidities that were significantly more common than the others in our COVID-pos patients were diabetes and chronic alcoholism. Both diseases decrease immune capacity and promote infections, ${ }^{23,24}$ which is entirely consistent given the context. The mortality rate in our study was particularly high: in our geriatric hospital, COVD-19 has doubled the patient mortality rate. First, the mortality rate for COVID-neg patients, which was $14.4 \%$ (over the month and a half of the study), was close to the mortality rate in the follow-up and rehabilitation units of the HUS geriatric hospital in 2017 , which was $10 \%$ (unpublished data). COVID-pos patients had a mortality rate of $29.2 \%$, which was equivalent to the data in residential institutions for dependent elderly people (EHPAD or retirement homes) in France where the mortality rate described is 25 to 33\% (unpublished data). In the same way in Italy and in China, the mortality rate is reported to have reached $34.5 \%$ in elderly people. $^{25}$

We have demonstrated also that OADs seem to diminish the mortality of elderly patients with COVID-19. Recent research analysed the interaction between SARS-CoV-2 proteins and human proteins. ${ }^{26}$ It appears that SARS-CoV-2 proteins probably interact with the mTORC1 (mammalian target of rapamycin complex 1) pathway. ${ }^{26}$ Metformin, which was the main OAD used in our diabetic COVID-pos patients (83.3\%), is an indirect inhibitor of mTORC1, and thus could explain the potential interest of metformin against COVID-19. ${ }^{26}$ Antipsychotics also decreased COVID-pos patient mortality: none of the 12 COVID-pos patients on antipsychotics died. As early as 1975, the first findings of the antiviral properties of antipsychotics were reported, first clinically, with the massive decrease of recurrences of genital herpes infections under chlorpromazine, and then biologically. ${ }^{27}$ Thus, phenothiazine and thiothixene compounds inhibit the replication of HSV 1 and 2, tick-borne encephalitis virus, Epstein-Barr virus, and measles virus. ${ }^{28}$ Haloperidol increases cell survival in the context of retroviral infection. ${ }^{29}$ In the same way, it has been demonstrated that the metabolites of clozapine, a so-called atypical antipsychotic, inhibit the replication of human immunodeficiency virus type 1 . The discovery of these two types of therapeutic classes (OADs and antipsychotics) should encourage us to test some of these drugs in therapeutic trials. We have therefore started a trial called COVID-Aging, dedicated solely to the elderly: this trial is adaptive, so that we will be able to include such drugs if one of the first drugs (hydroxychloroquine, azithromycin, or telmisartan) is inconclusive as to its potential to improve patient survival.

This study has methodological limitations. First, it was retrospective. Nevertheless, the fact that all data were accessible on professional medical software made it possible to better guarantee the completeness of the data. The second limitation concerns the use of RT-PCR: its sensitivity is most likely excellent, but the timing and depth of swabbing are limitations of this technique. To overcome this, the swabs were repeated in the COVID-neg group and when possible, a chest CT scan was 
performed. In only one case among 20 in the COVID-neg group was the CT scan consistent with COVID-19 infection.

Here we have shown for the first time that some specific treatments frequently used in the elderly in a geriatric context could have a preventive or curative effect. On the preventive side, PPIs seem to reduce the risk of infection with COVID-19 in a highly contagious context. Therefore, it seems important to keep patients on PPIs, including those taking them for no obvious reason, awaiting further studies in this area. On the curative side, OADs and antipsychotics seem to have beneficial effects on patient survival. In the latter case, it is obviously necessary for therapeutic trials to be carried out, and this must be done quickly in view of the major mortality rate in the elderly. Our finding regarding PPIs needs to be confirmed by further studies. If this were the case, PPIs would be very good candidates in view of their safety profile.

\section{Declaration of interests}

We declare no competing interests.

\section{Data sharing}

After publication, the data will be made available to others on reasonable requests to the corresponding author. A proposal with detailed description of study objectives and statistical analysis plan will be needed for evaluation of the reasonability of requests. Additional materials might also be required during the process of evaluation. Deidentified participant data will be provided after approval from the corresponding author.

\section{Acknowledgements}

We thank all patients and their families involved in the study, and all the staff of the Robertsau Geriatric Hospital in contact with patients, including interns, medical students, nurses, physiotherapists, psychologists, nurses' aides, housekeepers, as well as the hospital's support teams. We also thank Nick B. and Christian B. for their advice on the manuscript.

\section{References}

1. Zhu N, Zhang D, Wang W, et al. A Novel Coronavirus from Patients with Pneumonia in China, 2019. New England Journal of Medicine 2020; 382(8): 727-33.

2. Team TNCPERE. Vital Surveillances: The Epidemiological Characteristics of an Outbreak of 2019 Novel Coronavirus Diseases (COVID-19) -China, 2020. China CDC Weekly 2020; 2(8): 113-22.

3. Grasselli G, Zangrillo A, Zanella A, et al. Baseline Characteristics and Outcomes of 1591 Patients Infected With SARS-CoV-2 Admitted to ICUs of the Lombardy Region, Italy. JAMA 2020.

4. Europe WHO. COVID-19 weekly surveillance report, data of the week of 30 March - 5 April 2020 (Epi week 14/2020). http://wwweurowhoint/fr/health-topics/health-emergencies/coronaviruscovid-19/weekly-surveillance-report 2020; accessed April 10, 2020.

5. Richardson S, Hirsch JS, Narasimhan M, et al. Presenting Characteristics, Comorbidities, and Outcomes Among 5700 Patients Hospitalized With COVID-19 in the New York City Area. JAMA 2020.

6. Amanat F, Krammer F. SARS-CoV-2 Vaccines: Status Report. Immunity 2020. 
301 7. Bloch EM, Shoham S, Casadevall A, et al. Deployment of convalescent plasma for the 302 prevention and treatment of COVID-19. The Journal of Clinical Investigation 2020.

303 8. Vaduganathan M, Vardeny O, Michel T, McMurray JJV, Pfeffer MA, Solomon SD. Renin304 Angiotensin-Aldosterone System Inhibitors in Patients with Covid-19. N Engl J Med 2020.

305 9. Gurwitz D. Angiotensin receptor blockers as tentative SARS-CoV-2 therapeutics. Drug Dev Res 3062020.

307 10. Yang N, Shen H-M. Targeting the Endocytic Pathway and Autophagy Process as a Novel 308 Therapeutic Strategy in COVID-19. Int J Biol Sci 2020; 16(10): 1724-31.

309 11. Homolak J, Kodvanj I. Widely Available Lysosome Targeting Agents Should Be Considered as a 310 Potential Therapy for COVID-19. 2020.

311 12. Caruso D, Zerunian M, Polici M, et al. Chest CT Features of COVID-19 in Rome, Italy. 312 Radiology 2020: 201237.

313 13. WHO. https://www.who.int/docs/default-source/coronaviruse/real-time-rt-pcr-assays-forthe-detection-of-sars-cov-2-institut-pasteur-paris.pdf?sfvrsn=3662fcb6 2. 2020.

14. Hálfdánarson ÓÖ, Pottegård A, Björnsson ES, et al. Proton-pump inhibitors among adults: a nationwide drug-utilization study. Therapeutic Advances in Gastroenterology 2018; 11: 1756284818777943.

15. Hreinsson JP, Palsdóttir S, Bjornsson ES. The Association of Drugs With Severity and Specific Causes of Acute Lower Gastrointestinal Bleeding. Journal of clinical gastroenterology 2016; 50(5): 408-13.

16. Cetin $\mathrm{H}$, Wurm $\mathrm{R}$, Reichardt $\mathrm{B}$, et al. Increased risk of death associated with the use of proton pump inhibitors in dementia patients and controls - a pharmacoepidemiological claims data analysis. European Journal of Neurology; $\mathrm{n} / \mathrm{a}(\mathrm{n} / \mathrm{a})$.

17. Gomm W, von Holt K, Thomé F, et al. Association of Proton Pump Inhibitors With Risk of Dementia: A Pharmacoepidemiological Claims Data Analysis. JAMA Neurology 2016; 73(4): 410-6. 18. Lee $Y Y$, Jeon HK, Hong JE, et al. Proton pump inhibitors enhance the effects of cytotoxic agents in chemoresistant epithelial ovarian carcinoma. Oncotarget 2015; 6(33): 35040-50.

19. Spugnini EP, Baldi A, Buglioni S, et al. Lansoprazole as a rescue agent in chemoresistant tumors: a phase I/II study in companion animals with spontaneously occurring tumors. Journal of Translational Medicine 2011; 9(1): 221.

20. Simmons G, Reeves JD, Rennekamp AJ, Amberg SM, Piefer AJ, Bates P. Characterization of severe acute respiratory syndrome-associated coronavirus (SARS-CoV) spike glycoprotein-mediated viral entry. Proc Natl Acad Sci U S A 2004; 101(12): 4240-5.

21. Klompas M. Coronavirus Disease 2019 (COVID-19): Protecting Hospitals From the Invisible. Annals of Internal Medicine 2020.

22. Moatti J-P. The French response to COVID-19: intrinsic difficulties at the interface of science, public health, and policy. The Lancet Public Health 2020.

23. Geerlings SE, Hoepelman AI. Immune dysfunction in patients with diabetes mellitus (DM). FEMS Immunology \& Medical Microbiology 1999; 26(3-4): 259-65.

24. Mehta AJ, Yeligar SM, Elon L, Brown LA, Guidot DM. Alcoholism causes alveolar macrophage zinc deficiency and immune dysfunction. American journal of respiratory and critical care medicine 2013; 188(6): 716-23.

25. Chen T, Dai Z, Mo P, et al. Clinical characteristics and outcomes of older patients with coronavirus disease 2019 (COVID-19) in Wuhan, China (2019): a single-centered, retrospective study. The Journals of Gerontology: Series A 2020.

26. Gordon DE, Jang GM, Bouhaddou M, et al. A SARS-CoV-2-human protein-protein interaction map reveals drug targets and potential drug-repurposing. BioRxiv 2020.

27. Chang TW. Letter: Suppression of herpetic recurrence by chlorpromazine. N Engl J Med 1975; 293(3): 153-4.

28. Jones-Brando LV, Buthod JL, Holland LE, Yolken RH, Torrey EF. Metabolites of the antipsychotic agent clozapine inhibit the replication of human immunodeficiency virus type 1.

Schizophr Res 1997; 25(1): 63-70. 
353 29. Wunderlich V, Fey F, Sydow G. Antiviral effect of haloperidol on Rauscher murine leukemia 354 virus. Archiv fur Geschwulstforschung 1980; 50(8): 758-62.

355 
Figure 1: Probability of COVID-19 in elderly patients on proton pump inhibitors

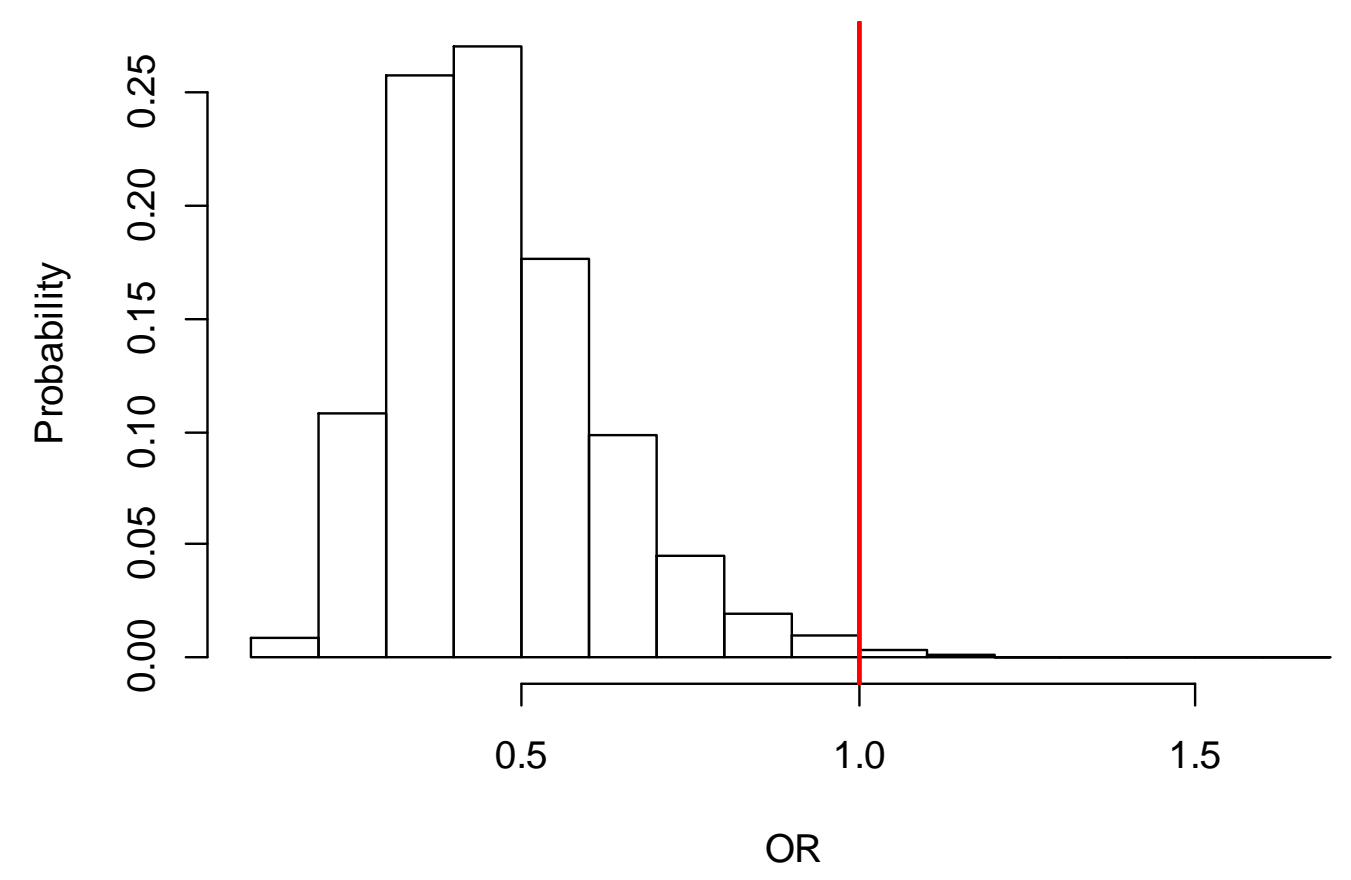




\section{patients}

COVID-19 patients Non COVID-19 patients Test statistic, $P$
$n=89$ $n=90$

\section{Age, years ${ }^{a}$}

Gender (F/M)

History $(\mathrm{Y} / \mathrm{N})$

High blood pressure
Diabetes
Smoking
Alcoholism
Heart disease
Atrial fibrillation
Chronic resp. failure
COPD
Dementia (AD, DLB, VD,
PDD, other)
Chronic kidney failure
Cancer (prostate, breast,
colon, lung, other)
Stroke
Depression
Peptic ulcer
Gastritis

Hiatus hernia, GERD

Number of SARS-CoV-2 RT-PCR -1,2,3,4,5-

Mortality rate $(\mathrm{N})^{\mathrm{c}}$

Recovery rate $(\mathrm{N})^{\mathrm{c}}$

Contact with COVID-19 -no/on site/other site/ home-

Viral compatible symptom (N) ${ }^{c, d}$

Symptoms (N) ${ }^{c}$

Dyspnoea
Cough $^{c}$
Oxygen therapy
Asthenia $^{c}$
Diarrhoea $^{c}$
Pain
Fall $^{c}$
Delirium $^{c}$

Lung auscultation -normal/rhonchi/bibasilar crackles/unilateral crackles/wheeze-

\section{Temperature $-{ }^{\circ} \mathrm{C}-\mathrm{b}$}

Pulse ${ }^{b}$

Systolic blood pressure - $\mathrm{mmHg}^{-}{ }^{b}$

Diastolic blood pressure $-\mathrm{mmHg}^{\mathrm{b}}$

Weight - kg- $^{b}$

Height $-\mathrm{cm}^{-}{ }^{\mathrm{b}}$

Obesity (N) ${ }^{c, e}$

Undernutrition $(\mathrm{N}) \mathrm{c}, \mathrm{f}$

Oxygen saturation -\%- $b$

Lung CT-scan -normal, suspected COVID-19, other pathology- $(\mathrm{N})$

Blood results ${ }^{b}$

\begin{tabular}{l} 
Leukocytes $-/ \mathrm{mm}^{3}$ - \\
Neutrophil polynuclear \\
cells $-/ \mathrm{mm}^{3}$ - \\
Lymphocytes $-/ \mathrm{mm}^{3}$ - \\
Platelets $-/ \mathrm{mm}^{3}$ - \\
\hline CRP $-\mathrm{mg} / \mathrm{l}-$ \\
Sodium $\left(\mathrm{Na}^{+}\right)-\mathrm{mmol} / \mathrm{l}-$ \\
Potassium $\left(\mathrm{K}^{+}\right)-\mathrm{mmol} / \mathrm{l}-$ \\
GFR $-\mathrm{ml} / \mathrm{min} / 1.73 \mathrm{~m}^{2}$ -
\end{tabular}

$\begin{array}{ll}84.4(7.9) & 83.8(8.6) \\ 58 / 31 & 64 / 26 \\ 61 / 28 & 66 / 24 \\ 36 / 53 & 21 / 69 \\ 19 / 70 & 14 / 76 \\ 13 / 76 & 4 / 86 \\ 53 / 36 & 53 / 37 \\ 42 / 47 & 36 / 54 \\ 9 / 80 & 17 / 73 \\ 6 / 83 & 9 / 81\end{array}$

$74(39,6,22,4,3) / 15 \quad 70(45,2,14,3,6) / 20$

$47 / 42 \quad 38 / 52$

$17(1,8,0,0,8) / 72 \quad 19(3,6,3,2,5) / 71$

$14 / 56 \quad 14 / 51$

$9 / 61 \quad 17 / 48$

$1 / 88 \quad 8 / 82$

$5 / 84 \quad 4 / 86$

$4 / 85 \quad 9 / 81$

$58,18,12,0,1$

$29.2 \%(26)$

$48.3 \%(43)$

$2 / 80 / 1 / 6$

$93.3 \%(83)$

$36.0 \%(32)$

$53.9 \%(48)$

$36.0 \%(32)$

$63.8 \%(51)$

$25.8 \%(23)$

$18.0 \%(16)$

$15.7 \%(14)$

$29.2 \%(26)$

$33 / 11 / 29 / 11 / 4$ (1 ND)

$37.8(1.3)$

$83(17)$

131 (23)

69 (14)

67 (18)

165 (9)

$22.6 \%(17)$

$28.0 \%(21)$

$93.6(4.8)$

$4 / 3 / 5(12)$

7057 (3979)

5232 (3984)

1118 (762)

249876 (98719)

$80.4(73.2)$

$138.5(4.9)$

$4.71(6.4)$

$68.48(24.30)$
$43,40,6,1,0$

$14.4 \%(13)$

$68.9 \%(62)$

6/70/3/10 (1 ND)

$67.8 \%(61)$

$32.2 \%$ (29)

$34.4 \%$ (31)

$17.8 \%$ (16)

$31.3 \%(25)$

$18.9 \%$ (17)

$14.4 \%(13)$

$22.2 \%(20)$

$22.2 \%(20)$

$48 / 16 / 12 / 10 / 4$

$37.4(1.1)$

83 (15)

132 (24)

69 (12)

$63(18)$

162 (10)

$14.5 \%$ (10)

$37.7 \%(26)$

93.9 (3.9)

$7 / 1 / 12(20)$

8722 (4802)

6535 (4327)

1317 (1009)

248070 (116758)

$69.5(76.8)$

137.9 (3.9)

$4.39(2.93)$

69.94 (35.68)
$\mathrm{F}=.043, \mathrm{P}=.836$

$\chi^{2}=.728, P=.425$

$\chi^{2}=.499, P=.513$

$\chi^{2}=6.040, P=.016^{*}$

$\chi^{2}=.999, P=.341$

$\chi^{2}=5.377, P=.023$ *

$\chi^{2}=.008, P=1.000$

$\chi^{2}=.941, P=.368$

$X^{2}=2.776, P=.137$

$\chi^{2}=.619, P=.591$

$\chi^{2}=6.058, P=.301$

$\chi^{2}=2.011, P=.179$

$\chi^{2}=6.980, P=.222$

$\chi^{2}=1.964, P=.580$

$\chi^{2}=4.825, P=.09$

$\chi^{2}=5.651, P=.034 *$

$\chi^{2}=.129, P=.747$

$\chi^{2}=2.014, P=.249$

$\chi^{2}=14.567, P=.006^{*}$

$\chi^{2}=8.480, P=.014 *$

$\chi^{2}=8.480, P=.014 *$

$\chi^{2}=5.661, P=.226$

$\chi^{2}=18.470, P<.0001 *$

$\chi^{2}=.278, P=.638$

$\chi^{2}=6.893 P=.011^{*}$

$\chi^{2}=7.534 P=.006 *$

$\chi^{2}=16.990, P<.0001 *$

$\chi^{2}=1.247 P=.286$

$\chi^{2}=.411 P=.549$

$\chi^{2}=1.226 P=.341$

$\chi^{2}=1.145 P=.309$

$\chi^{2}=12.938 P=.044$ *

$F=.359, P=.020$

$F=1.054, P=.306$

$\mathrm{F}=.896, \mathrm{P}=.345$

$F=1.397, P=.239$

$F=.034, P=.854$

$\mathrm{F}=.943, \mathrm{P}=.333$

$\chi^{2}=1.576 P=.285$

$\chi^{2}=1.532 P=.286$

$\mathrm{F}=3.125, P=.079$

$\chi^{2}=2.881 P=.237$

$\mathrm{F}=4.711, \mathrm{P}=.014^{*}$

$F=3.491, P=.041$ *

$\mathrm{F}=2.688, \mathrm{P}=.148$

$\mathrm{F}=.035, \mathrm{P}=.912$

$F=.026, P=.340$

$F=3.066, P=.306$

$F=.603, P=.679$

$F=1.543, P=.216$ 
and/or asthenia and/or diarrhoea. ${ }^{\mathrm{e}}$ Body mass index $>30 \mathrm{~kg} / \mathrm{m}^{2} .{ }^{\mathrm{f}}$ Body mass index $<21 \mathrm{~kg} / \mathrm{m}^{2}$.

$\mathrm{AD}=\mathrm{Alzheimer}$ 's disease; $\mathrm{COPD}=$ chronic obstructive pulmonary disease; $\mathrm{CRP}=\mathrm{C}$-reactive protein; $\mathrm{CT}$-scan=computed tomography scanner; $\mathrm{DLB}=\mathrm{dementia}$ with Lewy bodies; F=female; GERD=gastroesophageal reflux disease; GFR=glomerular filtration rate; $\mathrm{M}=$ male; $\mathrm{N}=\mathrm{no}$; $\mathrm{n}=$ number; PDD=Parkinson's disease dementia; $\mathrm{VD}=\mathrm{vascular}$ dementia; $\mathrm{Y}=\mathrm{yes}$. 


\section{Table 2: Probability of COVID-19 with or without each of the treatments}

The columns indicate the frequency of treatment in the sample, the odds-ratio -OR- (multiplication of the risk of COVID-19 when the patient has the treatment, as an a posteriori median and its $95 \%$ credibility interval), the estimated frequency of COVID-19 without treatment (median and 95\% CI), the estimated frequency of COVID-19 with treatment (median and $95 \% \mathrm{Cl}$ ) and the probability that the difference between the two frequencies ("with" and "without") is positive.

\begin{tabular}{|c|c|c|c|c|c|c|c|c|c|c|c|}
\hline & \multirow{2}{*}{$\begin{array}{l}\text { Treatment } \\
\text { frequency } \\
\text { (\%) }\end{array}$} & \multicolumn{3}{|c|}{ OR } & \multicolumn{3}{|c|}{$\begin{array}{c}\text { Probability of COVID-19 without } \\
\text { treatment }\end{array}$} & \multicolumn{3}{|c|}{$\begin{array}{l}\text { Probability of COVID-19 with } \\
\text { treatment }\end{array}$} & \multirow[t]{2}{*}{$\begin{array}{c}P \\
\text { (with }>\text { without) }\end{array}$} \\
\hline & & $50 \%$ & $2.5 \%$ & $97.5 \%$ & $50 \%$ & $2.5 \%$ & $97.5 \%$ & $50 \%$ & $2.5 \%$ & 97.5\% & \\
\hline ARBs & 15.08 & 1.3246 & 0.5813 & 2.9804 & 48.75 & 40.78 & 56.6 & 55.59 & 36.77 & 72.95 & .7514 \\
\hline ACE inhibitors & 21.79 & 1.6003 & 0.7931 & 3.3015 & 47.27 & 38.98 & 55.49 & 58.88 & 43.37 & 73.18 & .9029 \\
\hline Diuretics & 45.81 & 1.2192 & 0.6869 & 2.1713 & 47.34 & 37.79 & 57.32 & 52.38 & 41.66 & 62.95 & .7453 \\
\hline CCB & 22.35 & 0.782 & 0.3870 & 1.5718 & 51.09 & 42.85 & 59.34 & 45.04 & 30.42 & 60.15 & .2527 \\
\hline Beta-blockers & 40.78 & 1.2799 & 0.7085 & 2.3135 & 47.3 & 37.85 & 56.44 & 53.42 & 41.99 & 64.4 & .7909 \\
\hline Other antihypertensives & 6.15 & 0.8291 & 0.2319 & 2.7007 & 50.04 & 42.55 & 57.65 & 45.14 & 19.86 & 72.32 & .3771 \\
\hline Statins & 16.76 & 0.8723 & 0.3942 & 1.9177 & 50.36 & 42.3 & 58.21 & 46.82 & 29.98 & 64.35 & .3607 \\
\hline AD drugs & 3.35 & 0.9992 & 0.1939 & 5.1687 & 49.73 & 42.26 & 57.11 & 49.84 & 16.34 & 83.47 & .4995 \\
\hline PD drugs & 7.26 & 1.1984 & 0.3790 & 3.745 & 49.42 & 41.96 & 56.84 & 53.96 & 27.65 & 77.8 & .6224 \\
\hline Antidepressants & 18.44 & 1.0936 & 0.5162 & 2.3259 & 49.35 & 41.32 & 57.38 & 51.51 & 34.82 & 67.86 & .5907 \\
\hline Antipsychotics & 15.64 & 0.8583 & 0.3800 & 1.9028 & 50.33 & 42.5 & 58.19 & 46.53 & 29 & 64.52 & .3558 \\
\hline Anxiolytics & 32.4 & 1.2479 & 0.6685 & 2.3451 & 47.84 & 39.25 & 56.73 & 53.39 & 40.51 & 65.93 & .7537 \\
\hline Antiepileptics & 7.26 & 0.8517 & 0.2641 & 2.6427 & 49.99 & 42.36 & 57.57 & 45.99 & 21.34 & 71.8 & .3897 \\
\hline Antibiotics & 9.5 & 1.1494 & 0.4277 & 3.1204 & 49.44 & 41.91 & 56.99 & 52.97 & 30.14 & 74.82 & .6070 \\
\hline L-Thyroxin & 17.88 & 1.0114 & 0.4717 & 2.1574 & 49.63 & 41.77 & 57.78 & 50.01 & 33.07 & 66.63 & .5131 \\
\hline PPIs & 35.2 & 0.4381 & 0.2331 & 0.8175 & 56.78 & 47.6 & 65.45 & 36.58 & 25.5 & 48.75 & $.0053^{*}$ \\
\hline Paracetamol & 33.52 & 1.5143 & 0.8160 & 2.8415 & 46.33 & 37.35 & 55.19 & 56.56 & 44.16 & 68.64 & .9083 \\
\hline NSAIDs & 1.12 & 7.3082 & 0.4612 & 275.3777 & 49.36 & 42.02 & 56.74 & 87.53 & 31.4 & 99.63 & .9155 \\
\hline APT & 27.93 & 1.4209 & 0.7354 & 2.7624 & 47.23 & 38.92 & 55.85 & 55.98 & 42.17 & 69.28 & .8523 \\
\hline Anticoagulant & 42.46 & 0.8537 & 0.4746 & 1.5198 & 51.46 & 41.96 & 60.94 & 47.41 & 36.64 & 58.37 & .2981 \\
\hline Insulin & 12.29 & 2.3754 & 0.9676 & 6.4591 & 47.26 & 39.61 & 54.99 & 68.06 & 47.71 & 84.39 & .9711 \\
\hline OADs & 12.29 & 1.5376 & 0.6312 & 3.8643 & 48.44 & 40.62 & 56.34 & 58.94 & 38.42 & 77.59 & .8259 \\
\hline Corticosteroids & 5.59 & 0.6605 & 0.1735 & 2.3329 & 50.22 & 42.84 & 57.72 & 40.23 & 15.12 & 69.78 & .2617 \\
\hline Immunosuppressants & 1.12 & 7.379 & 0.4876 & 269.8301 & 49.65 & 42.34 & 57.08 & 87.85 & 32.52 & 99.62 & .9185 \\
\hline Potassium & 25.14 & 0.759 & 0.3840 & 1.4731 & 51.44 & 43.07 & 59.61 & 44.51 & 30.8 & 58.88 & .2074 \\
\hline Others & 64.8 & 0.7022 & 0.3781 & 1.2963 & 55.47 & 43.05 & 67.17 & 46.61 & 37.49 & 55.72 & .1301 \\
\hline
\end{tabular}

$\mathrm{AD}=$ =Alzheimer's disease; $\mathrm{ACE}=$ angiotensin-converting-enzyme inhibitors; $\mathrm{APT}=$ antiplatelet therapy; $\mathrm{ARBs=angiotensin} \mathrm{II} \mathrm{receptor} \mathrm{blockers;} \mathrm{CCB=calcium} \mathrm{channel} \mathrm{blockers;} \mathrm{NSAIDs=nonsteroidal} \mathrm{anti-inflammatory}$

drugs; OADs=oral antidiabetic drugs; PD=Parkinson's disease; PPIs=proton pump inhibitors. 